\title{
ASO Author Reflections: Acellular Dermal Matrix Reduces Myofibroblasts in Capsule Tissue
}

\author{
Il-Kug Kim, MD, $\mathbf{P h D}^{1}$ and Ung Sik Jin, $\mathrm{MD}, \mathbf{P h D}^{2}$ \\ ${ }^{1}$ Department of Plastic and Reconstructive Surgery, Yeungnam University College of Medicine, Daegu, Korea; \\ ${ }^{2}$ Department of Plastic and Reconstructive Surgery, Seoul National University College of Medicine, Seoul, Korea
}

\section{PAST}

Capsular contracture is a major complication of implantbased breast reconstruction. Recent studies have shown that use of acellular dermal matrices (ADM) for coverage of breast implants decreased or prevented capsular contractures. Moyer et al. $^{1}$ performed histopathological analysis of native capsules and ADM capsules in both nonirradiated and irradiated patients. They observed significant differences in cellular infiltration and elastin distribution only. Other researchers have also investigated the histological differences between subpectoral and ADM capsules and the mechanism by which the ADM reduces capsule formation around the breast implant. However, the mechanism remains unclear.

\section{PRESENT}

Although it is established that postmastectomy radiotherapy (PMRT) promotes capsular contracture, the effect of radiation on ADM capsule thickness has not been elucidated. In this study, there was no significant difference between the thickness of nonirradiated and irradiated ADM capsules. ${ }^{2}$ Myofibroblasts and macrophages, which play central roles in tissue fibrosis, were significantly reduced in

This ASO Author Reflections is a brief invited commentary on the article, "Inhibition Mechanism of Acellular Dermal Matrix on Capsule Formation in Expander-Implant Breast Reconstruction after Postmastectomy Radiotherapy," Ann Surg Oncol. 2018; 25: 2279-87.

(C) Society of Surgical Oncology 2018

First Received: 5 October 2018;

Published Online: 7 November 2018

U. S. Jin, MD, PhD

e-mail: usj1011@snu.ac.kr the ADM capsules compared with the subpectoral capsules. We first investigated the number of endothelial-mesenchymal transition (EndoMT) cells and vimentin positive fibroblasts, and the $T G F-\beta 1$ and $P D G F-B$ transcript levels in the capsular tissues. The number of these cells and transcript levels were also reduced in the ADM capsules. This study provides insight into capsule formation around a breast implant and how ADM inhibits capsular contracture in nonirradiated and irradiated tissues.

\section{FUTURE}

Various analyses using hematoxylin and eosin, $\alpha$ smooth muscle actin ( $\alpha$ SMA), vimentin, CD31, F4/80 expression, $\alpha \mathrm{SMA}$ and $\mathrm{CD} 31$ coexpression, and relative gene expression levels of $T G F-\beta 1$ and $P D G F-B$ were conducted in this study. It was difficult to include a large number of patients; therefore, only a small subset of the population was enrolled. A large-scale study on the mechanism of peri-implant capsule formation is needed. To confirm the suggested mechanism of capsule formation and its inhibition by ADM, well-controlled animal experiments are essential.

DISCLOSURE There are no conflicts of interests.

\section{REFERENCES}

1. Moyer HR, Pinell-White X, Losken A. The effect of radiation on acellular dermal matrix and capsule formation in breast reconstruction: clinical outcomes and histologic analysis. Plast Reconstr Surg. 2014;133:214-21.

2. Kim IK, Park SO, Chang H, Jin US. Inhibition mechanism of acellular dermal matrix on capsule formation in expander-implant breast reconstruction after postmastectomy radiotherapy. Ann Surg Oncol. 2018;25:2279-87. 